\title{
Clinical significance of the distribution of C4d deposits in different anatomic compartments of the allograft kidney
}

\author{
Ibrahim Batal $^{1}$, Alin Girnita ${ }^{1}$, Adriana Zeevi ${ }^{1}$, Bassel Abou Saab ${ }^{1}$, Sean Stockhausen ${ }^{1}$, \\ Ron Shapiro ${ }^{2}$, Amit Basu², Henkie Tan ${ }^{2}$, Claire Morgan ${ }^{2}$ and Parmjeet Randhawa ${ }^{1}$ \\ ${ }^{1}$ Department of Pathology, University of Pittsburgh Medical Center, Pittsburgh, PA, USA and ${ }^{2}$ Department of \\ Surgery, University of Pittsburgh Medical Center, Pittsburgh, PA, USA
}

\begin{abstract}
Diffuse C4d deposition in peritubular capillaries is a well-recognized marker of antibody-mediated rejection. The significance of staining patterns that are focal or affect non-peritubular capillary compartments is less well defined. Paired frozen section and paraffin-embedded tissue stains were performed in $\mathbf{5 2}$ kidney allograft biopsies, and correlated with clinicopathologic parameters. Diffuse peritubular capillary C4d deposits were more often seen in frozen sections (22/52, $43 \%$ frozen tissue vs $10 / 52,19 \%$ paraffin-embedded tissue), whereas focal staining was observed more frequently within paraffin sections $(13 / 52,25 \%$ paraffin-embedded tissue vs $7 / 52,14 \%$ frozen tissue). In biopsies taken from patients with a history of donor-specific antibodies, diffuse, focal and negative peritubular capillary C4d staining patterns were seen in 11/14 (79\%), 1/14 (7\%) and 2/14 (14\%) of frozen biopsies vs $5 / 14(36 \%), 6 / 14(43 \%)$ and $3 / 14(21 \%)$ of paraffin-embedded biopsies. Transplant glomerulopathy score in paraffin-embedded biopsies was higher in specimens with vs without glomerular basement membrane C4d staining (1.5 \pm 0.8 vs $1.0 \pm 0.6, P=0.03)$. Tubular basement membrane staining was present in $4 \%$ paraffin-embedded and $48 \%$ frozen specimens independent of tubular atrophy. Arteriolar hyalinosis score in paraffin-embedded specimens was higher in biopsies with vs those without arteriolar C4d deposits $(1.3 \pm 0.9$ vs $0.9 \pm 0.8, P=0.04)$. Arterial staining was unrelated to the degree of intimal thickening. In conclusion, peritubular capillary deposits correlate well with circulating donor-specific antibody. For paraffinembedded tissue, combining the results of focal and diffuse staining allows a diagnostic sensitivity comparable to diffuse staining in frozen tissue. Finally, C4d deposits preferentially in lesions of chronic transplant glomerulopathy and arteriolar hyalinosis.
\end{abstract}

Modern Pathology (2008) 21, 1490-1498; doi:10.1038/modpathol.2008.152; published online 26 September 2008

Keywords: C4d immunostain; antibody-mediated rejection; transplant glomerulopathy; allograft; kidney

Traditionally, the histologic diagnosis of antibodymediated rejection has been suggested based on peritubular capillary inflammation, thrombosis, fibrinoid necrosis, and demonstration of immunoglobulin and complement deposits in allograft tissue..$^{1-3}$ In recent years, the spectrum of pathologic changes associated with antibody-mediated rejection has expanded considerably. It is now well recognized that light microscopic findings can be quite subtle even in the presence of circulating

Correspondence: Dr P Randhawa, MD, Department of Pathology, E737 UPMC Montefiore Hospital, University of Pittsburgh, 3459 Fifth Avenue, Pittsburgh, PA 15213, USA or Dr A Girnita, MD, Department of Pathology, University of Pittsburgh Medical Center, University of Pittsburgh, 200 Lothrop St, W1558, Biomedical Science Tower, Pittsburgh, PA 15261, USA.

E-mail: randhawapa@upmc.edu or girnal@upmc.edu

Received 7 April 2008; revised 15 July 2008; accepted 19 July 2008; published online 26 September 2008 donor-specific antibodies. ${ }^{4-6}$ The conventional immunofluorescence panel of immunoglobulin and complement components has been replaced by the detection of C4d, a single complement degradation product that can independently predict unfavorable graft outcome. ${ }^{7-14} \mathrm{C} 4 \mathrm{~d}$ participates in the classic pathway of complement activation. Activated C4 is cleaved to C4a and C4b. C4b binds to an amino or hydroxyl group and is then converted to C4d, which can covalently bind with peritubular capillary basement membrane. ${ }^{15}$

Initial immunohistochemical methods for C4d utilized a monoclonal antibody and fresh frozen sections, which are not always available. In recent years, several medical centers have started using a polyclonal antibody that can be used to stain formalin-fixed paraffin-embedded tissue. Limited data are available on the comparative sensitivity of these two methods. Nadasady et al and Regele et al 
found formalin-fixed tissue to give acceptable results, ${ }^{16,17}$ whereas Seemayer et al ${ }^{18}$ indicated that formalin fixation had a detrimental effect on C4d detection. Most published articles on C4d immunohistochemistry have focused on peritubular capillary staining. The significance of glomerular basement membrane and tubular basement membrane staining is not yet well established. Sijpkens et $a l^{19}$ described an association between glomerular basement membrane staining and chronic transplant glomerulopathy. Bracamonte et $a l^{20}$ and Hever et $a l^{21}$ demonstrated tubular basement membrane deposits in association with BK virus nephropathy. We present our own experience with C4d immunohistochemistry in both fresh frozen and formalin-fixed paraffin-embedded tissues. Staining patterns of different anatomic locations were correlated with clinical and histologic parameters including the presence of circulating donor-specific antibodies. C4d staining was scored in a semiquantitative manner and correlated with histologic scores of chronic transplant glomerulopathy, tubular atrophy, arteriolar hyalinosis and arterial thickening using Banff criteria for renal allograft pathology.

\section{Materials and Methods}

\section{Case Selection}

Biopsies were selected based on availability of both frozen and paraffin sections for analysis. The routine practice in our medical center is to submit only formalin-fixed tissue. However, physicians do freeze a portion of the biopsy in selected cases, if there is clinical concern for antibody-mediated rejection.

\section{C4d Staining}

Paired frozen and formalin-fixed tissue C4d stains were performed on 52 kidney biopsies from 51 renal transplant recipients. Immunostaining in formalinfixed paraffin-embedded tissue was performed using rabbit anti-human C4d polyclonal antibody (Alpco Diagnostics, Windham, NH, USA). Briefly, $4 \mu \mathrm{m}$ sections of allograft kidney were deparaffinized, placed in a pressure cooker for $20 \mathrm{~min}$ and sequentially incubated at $37^{\circ} \mathrm{C}$ with TRIS-EDTA $(\mathrm{pH}$ 8.5), 1:50 dilution of primary antibody for $44 \mathrm{~min}$, 1:100 dilution of a biotinylated anti-rabbit secondary antibody for $8 \mathrm{~min}$, streptavidin-alkaline phosphate conjugate for $8 \mathrm{~min}$, Fast red A-naphthol for $8 \mathrm{~min}$, Fast red B for $8 \mathrm{~min}$ and a routine hematoxylin counterstain. All reagents were components of the Ventana Enhanced Alkaline Phosphatase Red Detection Kit (catalog no. 760-031; Ventana Medical Systems, Tucson, AZ, USA) and used according to the enclosed instructions.

The frozen tissue staining procedure used a mouse monoclonal antibody directed against human
C4d (Biogenesis, Kingston, NH, USA). Briefly, 6-8 $\mu \mathrm{m}$ frozen sections were obtained, dried at room temperature, fixed in cold acetone for $10 \mathrm{~min}$ and then placed into a $37^{\circ} \mathrm{C}$ incubator overnight. Morphosave bisulfate fixative $(10 \times)$ was used to help retain morphologic detail of friable tissue. The subsequent procedure was similar to paraffin-embedded staining except that the Alpco antibody was replaced by a 1:100 dilution of the Biogenesis antibody ( $32 \mathrm{~min}$ incubation at $\left.37^{\circ} \mathrm{C}\right)$.

\section{Interpretation of C4d Stain}

Evaluation of C4d staining was done primarily by the first author (IB), however, selected cases were jointly reviewed with the principal author (PR). Different anatomic compartments in the kidney were analyzed in both frozen and paraffin-embedded samples as follows:

(1) Linear or finely granular peritubular capillary staining was classified as diffuse when it was seen in more than $50 \%$ of microscopic fields available for evaluation. It was referred to as focal when present in $10-50 \%$ of biopsied tissue, and negative if the staining did not even reach that threshold. Areas with tubular atrophy were excluded from analysis. These criteria are in agreement with recommendations made in the 2007 Banff Conference.

(2) Glomerular basement membrane staining was evaluated according to the most intensely stained glomerulus. It was further classified as global, segmental and minimal when more than $50,10-50$ or less than $10 \%$ of glomerular capillaries were stained, respectively. It was considered negative when no glomerular basement membrane staining was observed.

(3) Tubular basement membrane staining was evaluated only within the cortex, as medullary tubules can be difficult to distinguish from the vasa recta by routine light microscopy. In individual tubules, staining was considered to be present if more than half of the tubular basement membrane circumference was stained. Staining was further characterized as diffuse, focal and negative when $>50,10-50$ and less than $10 \%$ of the tubules showed C4d deposits, respectively.

(4) Arteriolar staining was classified as diffuse, focal or negative, when $>50,10-50$ or $<10 \%$ of arterioles showed C4d deposits, respectively. Similar criteria were used for arterial staining evaluation. However, when only one artery was available for evaluation, the designations diffuse and focal reflected the percent of circumferential area of that vessel showing C4d deposits.

For each anatomic compartment, the total number of positive specimens was also determined by disregarding the actual pattern of staining. To facilitate 
comparison, C4d was graded on a semiquantitative scale (0-2) in the peritubular capillary, tubular basement membranes, arteriolar and arterial compartments of each biopsy. These scores corresponded to negative, focal and diffuse staining, respectively. For staining in the glomerular basement membrane, a four-point scale $(0-3)$ was assigned corresponding to negative, minimal, segmental or global staining, respectively.

\section{Histologic Evaluation}

Standard H\&E, PAS, silver and trichrome sections were evaluated according to Banff 97 criteria $^{1}$ as follows: chronic transplant glomerulopathy was scored according to the percentage of peripheral capillary loops showing double contour in the most severely affected glomerulus (cg0, cg1, cg2 and cg3). Tubular atrophy was graded as ct0, ct1, ct2 and ct3 according to the percentage of atrophic tubules in sampled cortical area. Arteriolar hyalinosis was scored according to the severity of PAS-positive hyaline thickness (ah0, ah1, ah2 and ah3). Chronic vascular thickening was graded according to the percentage of vascular narrowing in the most affected artery (cv0, cv1, cv2 and cv3). Histologic evaluation was performed on paraffin-embedded tissue biopsies as freezing artifacts make it difficult to evaluate chronic transplant glomerulopathy, tubular atrophy and arteriolar hyalinosis on frozen sections. Our analysis, therefore, assumes that the severity of histologic lesions as assessed on paraffinembedded tissue is applicable to the whole kidney (including the portion of this biopsy taken for frozen section C4d staining).

\section{Circulating Donor-Specific Antibodies}

Blood samples were collected on the day of transplantation and at multiple times after transplant. Serum/plasma specimens were tested by enzyme-linked immunosorbent assay (ELISA). We used commercial LAT-M ${ }^{\mathrm{TM}}$ and LAT-1288 ${ }^{\mathrm{TM}}$ ELISA kits (One Lambda, Canoga Park, CA, USA), in accord with the manufacturer's instructions to identify IgG anti-HLA class I and class II specific alloantibodies. Both IgG anti-HLA class I and class II specific antibodies were measured indirectly by a second enzyme-linked colorimetric reaction. The assay was read at $630 \mathrm{~nm}$ using an ELISA reader (ELX 800; BioTek, Winooski, Vermont, USA, and computer software from One Lambda). All tests were run in duplicate and the results of ELISA screen were expressed as a percent of the average positive serum control. Donor-specific antibodies were defined as antibodies directed against one or more donor HLA type. Positive donor-specific antibodies were detected in serum samples corresponding to $14 / 44$ $(32 \%)$ of these specimens. All positive circulating donor-specific antibodies were detected within 8 days of the biopsy except in two cases, one of them was performed 16 days before biopsy, whereas the other was positive 160 days before biopsy, following which it became negative, possibly due to allograft absorbance. $^{22}$

Clinical and pathologic parameters were obtained by retrospective blinded chart review in accordance with methods approved by the University of Pittsburgh Institutional Review Board (IRB protocol no. 602112).

\section{Statistics}

Rank sum tests (Kruskal-Wallis one-way analysis of variance on ranks and Mann-Whitney rank sum test) were used to compare C4d semiquantitative scores and staining patterns in different anatomic components. These statistical analyzes were performed using SigmaStat 2.0.3 software (SPSS Inc., Chicago, IL, USA). The ability of peritubular capillary staining to detect circulating donor-specific antibodies was evaluated using contingency tables and a $\chi^{2}$-test ( $P$-value expressed as Fisher's exact test). This statistical analysis was performed using Statistica (software from Statsoft, Tulsa, OK, USA, 1995). ${ }^{23} P$-values less than 0.05 were considered statistically significant.

\section{Results}

There were 52 kidney allograft specimens from 51 patients (29 men, 22 women) ranging in age between 19 and 81 years (mean $53 \pm 15$ years). The race of the patients was as follows: Caucasians $(n=36)$, African Americans $(n=9)$, American Indian $(n=1)$ and others or not available $(n=5)$. The sources of allografts were deceased donor $(n=30)$, living related donor $(n=13)$, living unrelated donor $(n=6)$ and not available $(n=2)$. The primary diseases that led to renal failure and transplantation were diabetic mellitus $(n=11)$, hypertension $(n=11)$, glomerulonephritis $(n=5)$, membranous glomerulopathy $(n=3)$, ureteral reflux $(n=3)$, cystic kidney diseases $(n=3)$, focal segmental glomerulosclerosis $(n=2)$, Alport syndrome $(n=1)$, cholesterol emboli $(n=1)$, interstitial nephritis $(n=1)$, pyelonephritis $(n=1)$, sarcoidosis $(n=1)$, Wegener's granuloma $(n=1)$, undetermined $(n=1)$ and not available $(n=6)$. The percentage of biopsies with detectable C4d staining together with C4d scores is presented in Table 1. Mean C4d scores for tubular basement membranes and arterioles were significantly higher in frozen compared with paraffinembedded biopsies. C4d staining intensity in all other compartments also tended be higher in frozen sections than paraffin-embedded samples, but the differences were not statistically significantly (Table 1).

A description of C4d staining patterns in individual anatomic compartments follows. 
Table 1 C4d staining patterns in different anatomic compartments of the allograft kidney

\begin{tabular}{|c|c|c|}
\hline & Frozen tissue & $\begin{array}{c}\text { Paraffin-embedde } \\
\text { tissue }\end{array}$ \\
\hline \multicolumn{3}{|c|}{ Peritubular capillaries } \\
\hline Positive & $29 / 52(56 \%)$ & $23 / 52(44 \%)$ \\
\hline Diffuse & $22 / 52(43 \%)$ & $10 / 52(19 \%)$ \\
\hline Focal & $7 / 52(14 \%)$ & $13 / 52(25 \%)$ \\
\hline Negative & $23 / 52(44 \%)$ & $29 / 52(56 \%)$ \\
\hline C4d score & $1.0 \pm 0.9$ & $0.6 \pm 0.8$ \\
\hline \multicolumn{3}{|c|}{ Glomerular basement membranes } \\
\hline Positive & $22 / 39(56 \%)$ & $27 / 48(56 \%)$ \\
\hline Global & $18 / 39(46 \%)$ & $12 / 48(25 \%)$ \\
\hline Segmental & $2 / 39(5 \%)$ & $6 / 48(12 \%)$ \\
\hline Minimal & $2 / 39(5 \%)$ & $9 / 48(19 \%)$ \\
\hline Negative & $17 / 39(44 \%)$ & $21 / 48(44 \%)$ \\
\hline C4d score & $1.5 \pm 1.5$ & $1.2 \pm 1.3$ \\
\hline \multicolumn{3}{|c|}{ Tubular basement membranes } \\
\hline Positive & $25 / 52(48 \%)$ & $2 / 52(4 \%)$ \\
\hline Diffuse & $5 / 52(10 \%)$ & $0 / 52(0 \%)$ \\
\hline Focal & $20 / 52(38 \%)$ & $2 / 52(4 \%)$ \\
\hline Negative & $27 / 52(52 \%)$ & $50 / 52(96 \%)$ \\
\hline C4d score ${ }^{a}$ & $0.6 \pm 0.7$ & $0.04 \pm 0.2$ \\
\hline \multicolumn{3}{|l|}{ Arterioles } \\
\hline Positive & $31 / 40(78 \%)$ & $34 / 48(71 \%)$ \\
\hline Diffuse & $29 / 40(73 \%)$ & $19 / 48(40 \%)$ \\
\hline Focal & $2 / 40(5 \%)$ & $15 / 48(31 \%)$ \\
\hline Negative & $9 / 40(22 \%)$ & $14 / 48(29 \%)$ \\
\hline C4d score ${ }^{b}$ & $1.5 \pm 0.9$ & $1.1 \pm 0.8$ \\
\hline \multicolumn{3}{|l|}{ Arteries } \\
\hline Positive & $19 / 29(65 \%)$ & $22 / 44(50 \%)$ \\
\hline Diffuse & $14 / 29(48 \%)$ & $18 / 44(41 \%)$ \\
\hline Focal & $5 / 29(17 \%)$ & $4 / 44(9 \%)$ \\
\hline Negative & $10 / 29(35 \%)$ & $22 / 44(50 \%)$ \\
\hline C4d score & $1.1 \pm 0.9$ & $0.9 \pm 1.0$ \\
\hline
\end{tabular}

A total of 52 biopsies were evaluated, but, as is apparent from the data presented, not all specimens had glomeruli, arteries, or arterioles available for evaluation in the tissue section stained for C4d.

${ }^{\mathrm{a}} P<0.01$ tubular basement membrane C4d score, frozen vs paraffinembedded biopsies (Mann-Whitney test).

${ }^{\mathrm{b}} P=0.028$ arteriolar C4d score, frozen vs paraffin-embedded biopsies (Mann-Whitney test).

\section{Peritubular Capillary Staining}

Within peritubular capillaries, the frequency of positive staining was somewhat higher in frozen compared to paraffin-embedded biopsies (56 vs $44 \%)$. Diffuse peritubular capillary C4d deposits had a tendency to be more common in frozen specimens (43 vs 19\%) whereas focal staining was observed somewhat more frequently in paraffinembedded biopsies (25\% paraffin embedded vs $14 \%$ frozen) (Table 1). Overall, there was a trend toward a higher C4d staining scores in frozen (1.0 \pm 0.9$)$ compared with paraffin-embedded biopsies $(0.6 \pm 0.8)(P=0.086)$.

Information regarding contemporaneous circulating donor-specific antibody was available for $44 / 52$ allograft kidney biopsies from 43 patients (Table 2). For frozen biopsies obtained from patients with a
Table 2 The proportion of circulating donor-specific antibodies with different peritubular capillary staining patterns in frozen tissue and paraffin-embedded tissue

\begin{tabular}{lcr}
\hline & $\begin{array}{c}\text { DSA present } \\
(\mathrm{n}=14)\end{array}$ & $\begin{array}{c}\text { DSA absent } \\
(\mathrm{n}=30)\end{array}$ \\
\hline $\begin{array}{l}\text { Frozen sections } \\
\text { Negative }\end{array}$ & $2(14 \%)$ & $18(60 \%)$ \\
$\quad$ Focal & $1(7 \%)$ & $6(20 \%)$ \\
Diffuse & $11(79 \%)^{\mathrm{a}}$ & $6(20 \%)$ \\
& & \\
Paraffin-embedded tissue & $3(21 \%)$ & $23(77 \%)$ \\
$\quad$ Negative & $6(43 \%)^{\mathrm{b}}$ & $4(13 \%)$ \\
Focal & $5(36 \%)^{\mathrm{b}}$ & $3(10 \%)$ \\
Diffuse & $11(79 \%)^{\mathrm{c}}$ & $7(23 \%)$ \\
$\quad$ All positive biopsies & & \\
\hline
\end{tabular}

DSA, donor-specific antibodies.

aPrevalence of DSA in diffuse C4d stain pattern is significantly higher than focal and negative pattern of staining in frozen biopsies (diffuse $v s$ focal, $P=0.02$ ) and (diffuse vs negative, $P=0.0005$ ).

${ }^{b}$ Prevalence of DSA in focal and diffuse C4d stain patterns are significantly higher compared to negative staining in paraffinembedded biopsies (diffuse vs negative, $P=0.003$ ) and (focal vs negative, $P=0.002$ ).

${ }^{\mathrm{C}}$ The prevalence of DSA in paraffin-embedded biopsies when both diffuse $(36 \%)$ and focal $(43 \%)$ staining are considered together (all positive paraffin-embedded biopsies $79 \%$ ) is identical to the prevalence of DSA in diffuse C4d staining specimens in FS (79\%).

history of circulating donor-specific antibodies $(n=14)$, diffuse C4d peritubular capillary staining was seen in 11/14 (79\%) (MHC type II $(n=8)$, MHC type I $(n=3))$, whereas focal and negative staining were found in $1 / 14(7 \%)$ (MHC type II $(n=1))$ and $2 / 14(14 \%)$ (MHC type I $(n=1)$, both types $(n=1))$ biopsies, respectively. Within paraffin-embedded specimens obtained from the same patients, diffuse staining was present in 5/14 (36\%) (MHC type II $(n=4)$, MHC type I $(n=1))$, whereas focal and negative staining were present in 6/14 (43\%) (MHC type II $(n=3)$, MHC type I $(n=2)$, both types $(n=1))$ and $3 / 14(21 \%)$ (MHC type II $(n=2)$, MHC type I $(n=1)$ ) biopsies, respectively. Thus, in frozen biopsies, diffuse peritubular capillary staining was associated more often with the presence of circulating donor-specific antibodies compared to focally and negatively stained biopsies (diffuse vs negative, $P=0.0005$; diffuse $v s$ focal, $P=0.02$ ). The frequency of donor-specific antibodies in focal vs negative C4d stained biopsies did not reach statistical significance in this small data set. In contrast to frozen specimens, focal staining in paraffin-embedded biopsies (Figure 1) seemed to be associated with donor-specific antibodies as frequently as diffuse staining (diffuse $v s$ negative staining, $P=0.003$; focal vs negative 0.002; diffuse vs focal, no significant difference). Combining diffuse 5/14 (36\%) and focal 6/14 (43\%) staining in paraffin-embedded biopsies allowed documentation of peritubular capillary deposits in 11/14 (79\%) biopsies with donor-specific antibodies (Table 2). This latter frequency was similar to that attained by diffuse 
peritubular capillary staining in frozen tissue (11/14 $(79 \%))$. Within the limits of the sample size examined, the proportion of C4d-positive biopsies was similar in patients with MHC class I and class II circulating donor-specific antibodies in both frozen (3/5 MHC I vs 9/10 MHC II) and paraffin-embedded samples (4/5 MHC I vs 8/10 MHC II).

Circulating donor-specific antibodies were absent in 6/17 (35\%) of frozen specimens with diffuse peritubular capillary staining and $6 / 7(86 \%)$ of specimens with focal staining. In paraffin-embedded samples, absence of circulating donorspecific antibodies was noted in $3 / 8(38 \%)$ and $4 / 10(40 \%)$ of samples with diffuse and focal peritubular capillary staining, respectively. To evaluate the clinical significance of this apparently high rate of false-positive C4d stains, we separately analyzed patients with diffuse $\mathrm{C} 4 \mathrm{~d}$ and the presence (group A, $n=11$ ), or absence (group B, $n=6$ ) of circulating donor-specific antibodies. Group A patients had an inferior outcome comparable to group B patients. Specifically, 6/11 patients within group A developed graft failure due to chronic allograft nephropathy $(n=5)$ or refractory rejection $(n=1)$,

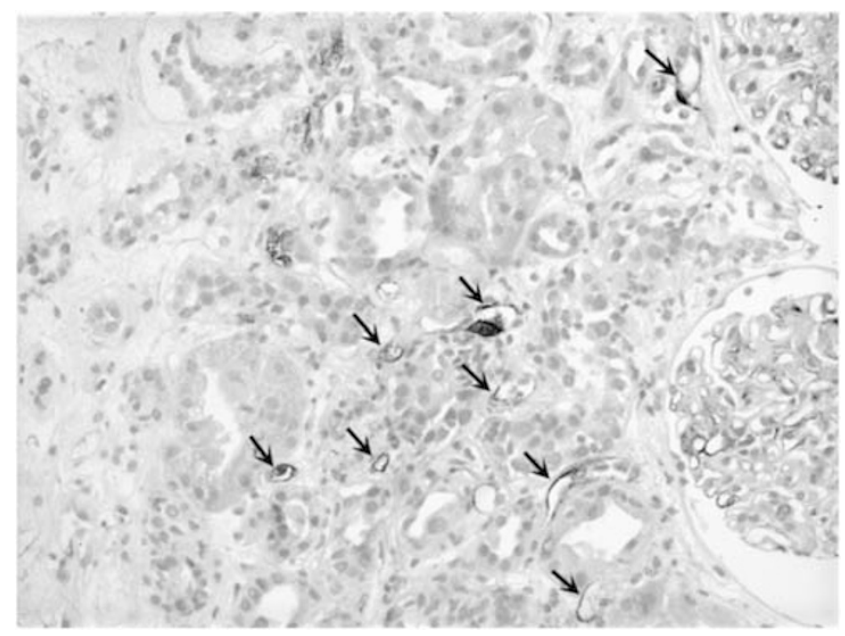

Figure 1 Focal peritubular capillary deposits of C4d in a patient with demonstrable circulating donor-specific antibodies (polyclonal C4d staining performed on formalin-fixed paraffinembedded tissue, original magnification $\times 200$ ). whereas 3/6 patients within group B developed graft failure due to chronic allograft nephropathy $(n=2)$ and recurrent IgA nephropathy $(n=1)$. There was a trend toward earlier detection of diffuse C4d staining in group A compared to group B (458 \pm 723 vs $2190 \pm 2486$ days after transplantation). The onset of graft failure following first detection of diffuse C4d was more rapid in group A $(80 \pm 81$ vs $276 \pm 102$ days, $P=0.03$ ). The length of clinical follow-up was not significantly different between these two groups (413 \pm 467 days group A vs $307 \pm 86$ days group B).

\section{Glomerular Basement Membrane}

In frozen tissue, glomeruli were available in 39 biopsies (Table 1). C4d staining pattern was global in $46 \%$, segmental in $5 \%$, minimal in $5 \%$ and negative in $44 \%$ of samples. In 48 of paraffin-embedded specimens with glomeruli available for evaluation, the staining pattern was similar, with global, segmental, minimal and negative staining found in 25, 12, 19 and 44\%, respectively. Glomerular basement membrane C4d score in frozen and paraffinembedded groups $(1.5 \pm 1.5$ frozen vs $1.2 \pm 1.3$ paraffin embedded) was also not significantly different. To assess a potential relationship between transplant glomerulopathy and C4d deposition, we assigned cg scores to paraffin-embedded biopsies whereas different patterns of C4d deposition in the glomerular basement membranes were evaluated in both frozen and permanent samples (Table 3). In paraffin-embedded biopsies, the mean cg score for all biopsies with C4d deposits was higher than the corresponding score for C4d-negative biopsies $(1.5 \pm 0.8$ vs $1.1 \pm 0.8, P=0.03$; Figure 2). In frozen tissue, this difference did not reach statistical significance $(1.6 \pm 0.8$ vs $1.1 \pm 0.8, P=0.1)$. In paraffin-embedded biopsies with positive glomerular membrane staining, cg scores tended to be the highest with a global staining pattern $(1.7 \pm 0.8)$, and the lowest in biopsies with minimal staining $(1.3 \pm 0.9)$. This relationship was less obvious in frozen sectioned specimens. All four biopsies with severe chronic transplant glomerulopathy (grade cg3) showed C4d deposits within the glomerular

Table 3 Distribution of chronic transplant glomerulopathy scores in biopsies classified by pattern of C4d deposition in glomerular basement membranes

\begin{tabular}{|c|c|c|c|c|c|}
\hline & Global & Segmental score & Minimal & Negative & All positive biopsies \\
\hline $\begin{array}{l}\text { Biopsies with GBM deposits in } \\
\text { FS }(n=39)\end{array}$ & $1.6 \pm 0.8(n=18)$ & $1.5 \pm 0.7(n=2)$ & $2.0 \pm 1.4(n=2)$ & $1.1 \pm 0.8(n=17)$ & $1.6 \pm 0.8(n=22)$ \\
\hline $\begin{array}{l}\text { Biopsies with GBM deposits in } \\
\operatorname{PE}^{\mathrm{a}}(n=48)\end{array}$ & $1.7 \pm 0.8(n=12)$ & $1.5 \pm 0.8(n=6)$ & $1.3 \pm 0.9(n=9)$ & $1.0 \pm 0.6(n=21)$ & $1.5 \pm 0.8(n=27)$ \\
\hline
\end{tabular}

FS, frozen section; PE, paraffin embedded; GBM, glomerular basement membrane; cg, chronic transplant glomerulopathy score.

${ }^{\mathrm{a}} P=0.03$ (cg score all positive vs negative staining), Mann-Whitney test.

cg scores presented as mean \pm s.d. (no of biopsies).

For C4d assessment, intact glomeruli in well-preserved areas were available in 48 paraffin-embedded specimens and in 39 frozen specimens. Scores for cg were assessed on silver stained paraffin-embedded sections only. 


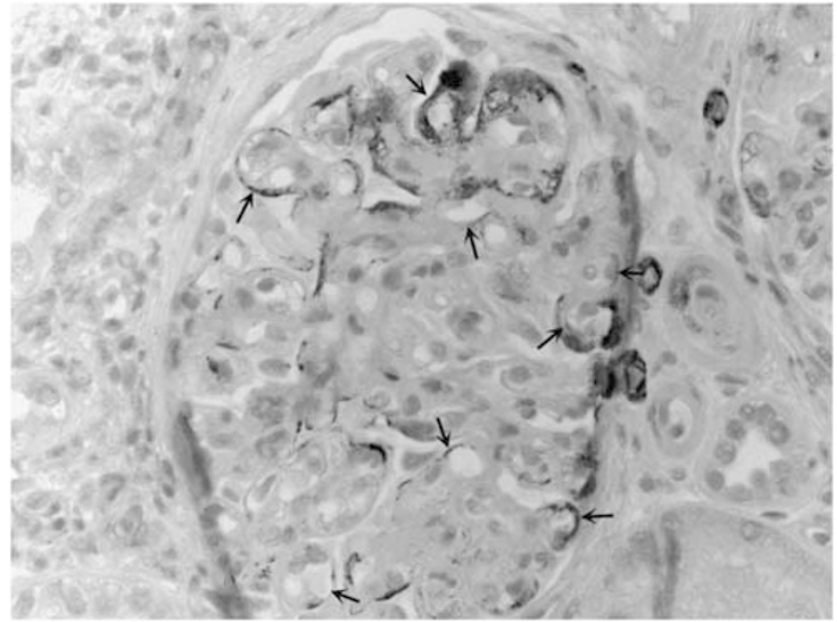

Figure 2 Glomerulus with chronic transplant glomerulopathy showing segmental C4d deposits in capillary basement membranes (polyclonal C4d staining performed on formalin-fixed paraffin-embedded tissue, original magnification $\times 600$ ).

basement membranes in both frozen specimens (global $(n=3)$ and minimal $(n=1))$ and paraffinembedded specimens (global $(n=2)$, segmental $(n=1)$ and minimal $(n=1))$.

\section{Tubular Basement Membrane}

C4d deposits in the tubular basement membrane were seen in $48 \%$ of frozen biopsies, and could be further characterized as diffuse and focal in 10 and $38 \%$, respectively. In contrast, only $4 \%$ of paraffinembedded biopsies showed staining for C4d and both biopsies in this category had only focal deposits. Thus, C4d staining score was significantly higher in frozen compared to paraffin-embedded biopsies ( $0.6 \pm 0.7$ vs $0.04 \pm 0.2, P<0.001$; Table 1$)$. The mean tubular atrophy score (ct) was similar in biopsies with and without C4d deposits in the tubular basement membranes $(1.8 \pm 0.9$ vs $1.5 \pm 0.9$, frozen samples; Table 4). The tubular atrophy score was also comparable in specimens with diffuse and focal staining pattern. One of the two paraffinembedded biopsies that had tubular basement membrane staining demonstrated BK virus nephropathy, and in this case, tubular basement membrane staining was accompanied by parietal Bowman's capsular epithelial basement membrane staining (Figure 3).

\section{Vascular Staining}

Arterioles were seen in 40 frozen specimens and the C4d stain was interpreted as diffuse, focal and negative in 73,5 and $22 \%$, respectively. In 48 paraffin-embedded biopsies, the distribution of C4d was diffuse and focal in 40 and $31 \%$, respectively, whereas the remaining $29 \%$ of biopsies were negative. The intensity of C4d staining was higher

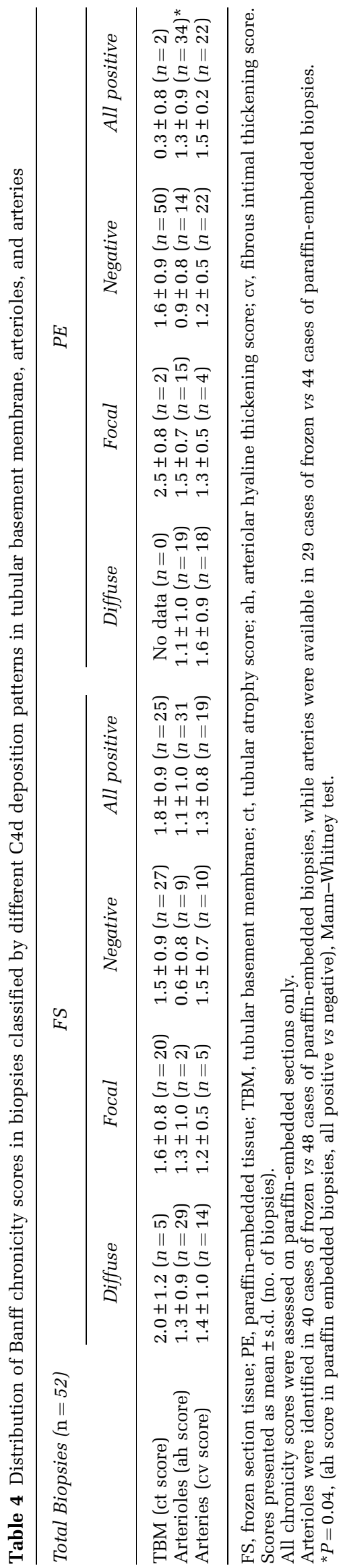

Modern Pathology (2008) 21, 1490-1498 
1496
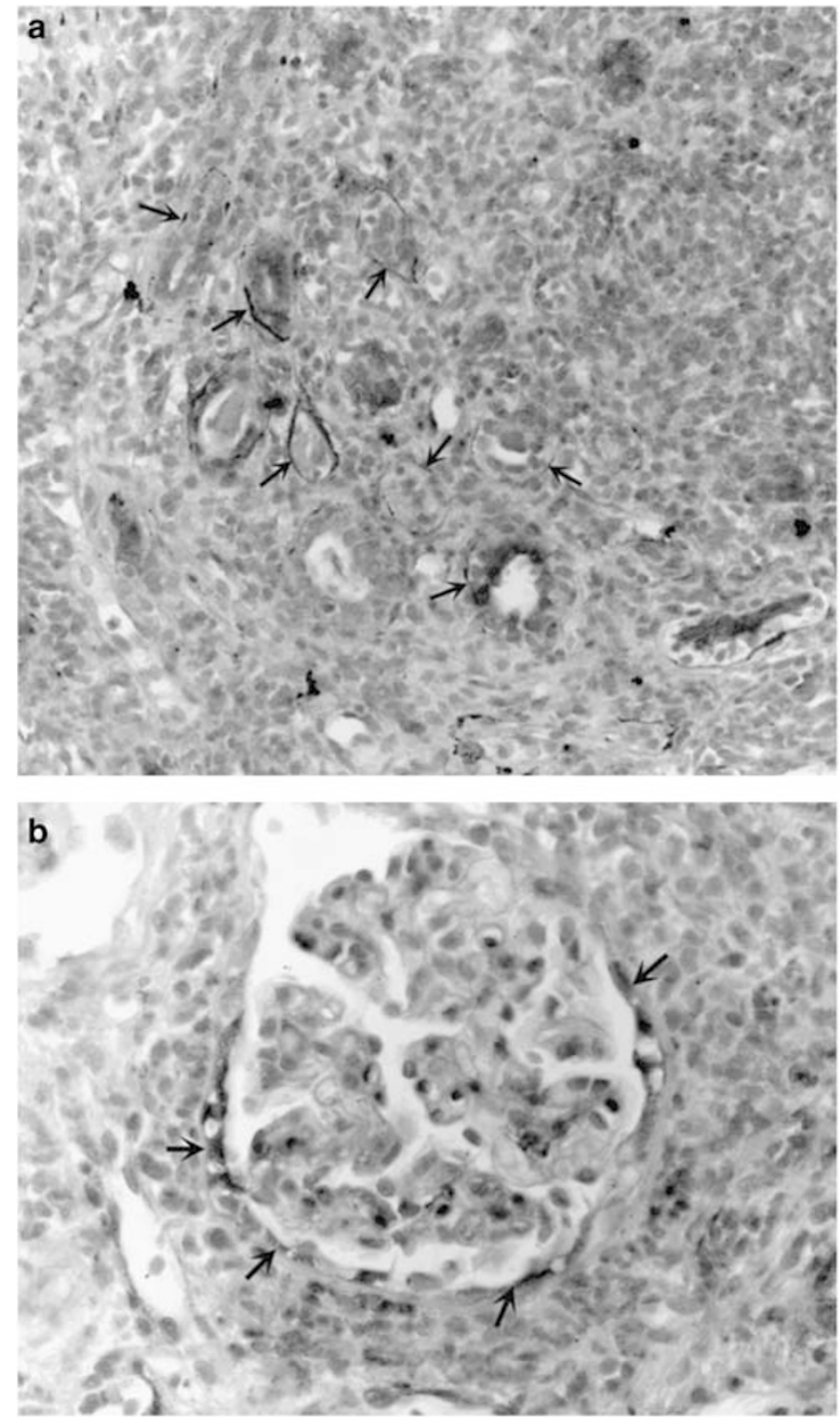

Figure 3 C4d staining along the tubular basement membranes (a) and Bowman's capsule epithelium (b) in the case of BK nephropathy (polyclonal C4d staining performed on formalinfixed paraffin-embedded tissue, original magnification $\times 200(\mathbf{a})$ and $\times 400(\mathbf{b})$.

in frozen than paraffin-embedded specimens $(1.5 \pm 0.9$ vs $1.1 \pm 0.8, P=0.028$; Table 1$)$. In paraffin-embedded specimens, biopsies with positive C4d staining showed higher arteriolar hyalinosis scores compared to negatively stained biopsies $(1.3 \pm 0.9$ vs $0.9 \pm 0.8, \quad P=0.04$; Table 4). This relationship was less obvious in frozen specimens $(1.1 \pm 1.0$ all positively stained vs $0.6 \pm 0.8$ negatively stained, no significant difference; Table 4), possibly because frozen sections typically contained very small amounts of renal parenchyma with only one or two arterioles available for evaluation. Arteries were observed in 29 frozen and 44 paraffin-embedded specimens and showed a similar prevalence of C4d staining (65\% frozen vs $50 \%$ paraffin embedded; Table 1). The staining pattern characterized as focal or diffuse was also similar in both specimen groups. Arterial staining appeared to be independent of the degree of arterial intimal thickening (cv score; Table 4).

\section{Discussion}

Several observations made during the course of this study call for comment. First, we found peritubular capillaries stain more often and more diffusely in frozen compared to paraffin-embedded tissue. However, in paraffin-embedded biopsies, if focal and diffuse staining patterns are combined, the association with circulating donor-specific antibodies is comparable to that attained by diffuse peritubular capillary staining in frozen biopsies. Before this work, three other studies have compared the relative sensitivity of C4d peritubular capillary staining using polyclonal antibodies on paraffin-embedded tissue and monoclonal antibodies on frozen tissue. Regele et $a l^{17}$ found peritubular capillary C4d deposits in 5/12 (42\%) biopsies using either method, and concluded that there was no difference in staining. Nadasdy et $a l^{16}$ state that there was no difference in staining pattern between frozen and paraffin-embedded tissues. However, on perusal of their tabulated data, it becomes apparent that $4 / 17$ biopsies that showed diffuse C4d on frozen tissue, gave only a focal reaction on paraffin-embedded tissue, whereas another biopsy showed complete loss of peritubular capillary staining. Seemayer et $a l^{18}$ noted diffuse and focal $\mathrm{C} 4 \mathrm{~d}$ peritubular capillary staining in 40 and $36 \%$ of frozen vs 30 and $16 \%$ of paraffin-embedded samples, respectively, and concluded that C4d staining in frozen tissue is more sensitive than paraffin-embedded tissue. With regard to the association between C4d staining and detection of donor-specific antibodies, Nadasdy et al found that 9/14 (64\%) of diffuse and $1 / 2(50 \%)$ of focal staining in frozen biopsies were associated with donor-specific antibodies, whereas in paraffin-embedded biopsies, 7/10 (70\%) diffuse and $2 / 5(40 \%)$ focal staining reactions were associated with these antibodies. In our study, considering biopsies taken from patients with a history of donor-specific antibodies, diffuse, focal and negative peritubular capillary C4d staining patterns were seen in $11 / 14(79 \%), 1 / 14(7 \%)$ and $2 / 14(14 \%)$ of frozen $v s 5 / 14(36 \%), 6 / 14(43 \%)$ and $3 / 14(21 \%)$ of paraffin-embedded biopsies. The absence of detectable circulating donor-specific antibodies in some patients with C4d-positive biopsies has been noted before, and even more frequently than observed by us. Thus, Worthington et a ${ }^{13}$ found that $9 / 14$ (64\%) of their biopsies with diffuse peritubular capillary C4d staining and all their focally stained biopsies $(100 \%)$ were not associated with circulating donorspecific antibodies. Similarly, Lederer et al $l^{8}$ found that $11 / 25(44 \%)$ of specimens with positive peritubular capillary C4d staining were not associated with donor-specific antibodies. Potential 
explanations include technical issues, different approaches for peritubular capillary C4d staining evaluation and scoring, complete absorbance of high affinity antibodies by allograft tissue ${ }^{22}$ and non-HLA antibodies, which would not be detected during routine testing of donor-specific antibody. ${ }^{24}$ In our study, the latter two possibilities are supported by the fact that diffuse C4d was associated with inferior outcome even in the absence of demonstrable donorspecific antibodies (three of six grafts lost). Indeed, in view of this unfavorable graft outcome, one could question whether the term 'false' positive is even appropriate for these cases.

According to some investigators, C4d deposits within glomerular basement membrane are observed in frozen but not paraffin-embedded specimens. In our laboratory, glomerular basement membrane staining is commonly found in paraffin-embedded biopsies. Regele et $a l^{17}$ described glomerular basement membrane C4d stain in 12/12 of frozen but only $2 / 12$ of paraffin-embedded biopsies. In another study, they demonstrated glomerular basement membrane staining in $6 \%$ of paraffin-embedded general kidney transplant biopsies and $12 \%$ of paraffin-embedded biopsies with chronic transplant glomerulopathy. ${ }^{25}$ Sijpkens et $a l^{19}$ found C4d depositions in glomerular capillaries to be both sensitive and specific for transplant glomerulopathy when they observed glomerular C4d deposits in $10 / 11(91 \%)$ of biopsies with transplant glomerulopathy vs $2 / 13$ (15\%) of biopsies without transplant glomerulopathy. Conversely, Seemayer et $a l^{18}$ found C4d staining to be a nonspecific indicator of glomerular capillary damage. However, no formal quantitation of $\mathrm{cg}$ was performed in their study. In our study, we found significant higher cg score in paraffin-embedded biopsies that were associated with positive glomerular basement membrane staining $(P=0.03)$ as well as a trend toward a significant difference in frozen specimens with glomerular staining $(P=0.1)$. In our opinion, glomerular basement membranes staining for C4d should not be regarded as specific for transplant glomerulopathy, as we have seen this finding in allograft kidneys with recurrent membranous nephropathy, membranoproliferative glomerulonephritis and anti-glomerular basement membrane nephritis (unpublished observation).

To the best of our knowledge, no previous study has compared tubular basement membrane staining in frozen and paraffin-embedded material. We found C4d tubular basement membrane staining primarily in frozen tissue, but do not understand the reason for this difference, although it may reflect the stability of the antigenic epitopes recognized by the antibodies used for immunohistochemistry. Seemayer et $a l^{18}$ noted that tubular basement membrane staining for C4d localizes to areas of tubular atrophy, but, no formal quantization of ct score was performed in their study. In our biopsy material, tubular staining for C4d on frozen speci- mens did not correlate with the Banff score for tubular atrophy. Another pertinent study with respect to tubular basement membrane staining for C4d is that of Bracamonte et al, ${ }^{20}$ who described granular tubular basement membrane immune deposits in 16/30 (53\%) of patients showing BK virus nephropathy. In our material, tubular basement membrane staining for C4d could not be related to BK virus infection in frozen sections. However, one out of the two paraffin-embedded biopsies with this finding did, indeed, have viral nephropathy. Of interest, in this latter biopsy Bowman's capsular C4d staining was also observed. As BK virus can infect the Bowman's capsular epithelium,,$^{26}$ one could speculate that this finding reflects virus-induced complement activation at this anatomic location.

Finally, we found arteriolar C4d to be more often diffuse and associated with a higher score in frozen compared to paraffin-embedded tissue (Table 1). This may be due to an increase in sensitivity when staining is performed on frozen tissue. Arteriolar C4d deposition is also commonly found in liver, intestine and heart allografts and does not seem to correlate with a diagnosis of antibody-mediated rejection (unpublished observations). One could speculate that this represents complement activation following microvascular injury because of hypertension or calcineurin inhibitor toxicity. The fact that such injury does not occur in more proximal vessels might explain the lack of correlation between intimal thickening and C4d deposition in small- to medium-sized arteries.

\section{Acknowledgements}

We thank Cary Sipos from the Immunohistochemistry Laboratory, University of Pittsburgh Medical Center for technical assistance. This work was supported by grants NIH R01-51227 and NIH RO1063360.

\section{Disclosure/conflict of interest}

None.

\section{References}

1 Racusen LC, Solez K, Colvin RB, et al. The Banff 97 working classification of renal allograft pathology. Kidney Int 1999;55:713-723.

2 Shimizu A, Colvin RB. Pathological features of antibody-mediated rejection. Curr Drug Targets Cardiovasc Haematol Disor 2005;5:199-214.

3 Mauiyyedi S, Colvin RB. Humoral rejection in kidney transplantation: new concepts in diagnosis and treatment. Curr Opin Nephrol Hypertens 2002;11:609-618. 
4 Scornik JC, LeFor WM, Cicciarelli JC, et al. Hyperacute and acute kidney graft rejection due to antibodies against B cells. Transplantation 1992;54:61-64.

5 Russ GR, Nicholls C, Sheldon A, et al. Positive B lymphocyte crossmatch and glomerular rejection in renal transplant recipients. Transplant Proc 1987;19:785-788.

6 Collins AB, Schneeberger EE, Pascual MA, et al. Complement activation in acute humoral renal allograft rejection: diagnostic significance of C4d deposits in peritubular capillaries. J Am Soc Nephrol 1999;10:2208-2214.

7 Herzenberg AM, Gill JS, Djurdjev O, et al. C4d deposition in acute rejection: an independent long-term prognostic factor. J Am Soc Nephrol 2002;13:234-241.

8 Lederer SR, Kluth-Pepper B, Schneeberger H, et al. Impact of humoral alloreactivity early after transplantation on the long-term survival of renal allografts. Kidney Int 2001;59:334-341.

9 Feucht HE, Schneeberger H, Hillebrand G, et al. Capillary deposition of C4d complement fragment and early renal graft loss. Kidney Int 1993;43:1333-1338.

10 Colvin RB. Antibody-mediated renal allograft rejection: diagnosis and pathogenesis. J Am Soc Nephrol 2007;18:1046-1056.

11 Racusen LC, Colvin RB, Solez K, et al. Antibodymediated rejection criteria-an addition to the Banff 97 classification of renal allograft rejection. Am J Transplant 2003;3:708-714.

12 Feucht HE, Mihatsch MJ. Diagnostic value of C4d in renal biopsies. Curr Opin Nephrol Hypertens 2005;14:592-598.

13 Worthington JE, McEwen A, McWilliam LJ, et al. Association between C4d staining in renal transplant biopsies, production of donor-specific HLA antibodies, and graft outcome. Transplantation 2007;83:398-403.

14 Nickeleit V, Andreoni K. The classification and treatment of antibody-mediated renal allograft injury: where do we stand? Kidney Int 2007;71:7-11.

15 Campbell RD, Gagnon J, Porter RR. Amino acid sequence around the thiol and reactive acyl groups of human complement component C4. Biochem J 1981;199:359-370.
16 Nadasdy GM, Bott C, Cowden D, et al. Comparative study for the detection of peritubular capillary C4d deposition in human renal allografts using different methodologies. Hum Pathol 2005;36:1178-1185.

17 Regele H, Exner M, Watschinger B, et al. Endothelial C4d deposition is associated with inferior kidney allograft outcome independently of cellular rejection. Nephrol Dial Transplant 2001;16:2058-2066.

18 Seemayer CA, Gaspert A, Nickeleit V, et al. C4d staining of renal allograft biopsies: a comparative analysis of different staining techniques. Nephrol Dial Transplant 2007;22:568-576.

19 Sijpkens YW, Joosten SA, Wong MC, et al. Immunologic risk factors and glomerular C4d deposits in chronic transplant glomerulopathy. Kidney Int 2004;65: 2409-2418.

20 Bracamonte E, Leca N, Smith KD, et al. Tubular basement membrane immune deposits in association with BK polyomavirus nephropathy. Am J Transplant 2007;7:1552-1560.

21 Hever A, Nast CC. Polyoma virus nephropathy with simian virus 40 antigen-containing tubular basement membrane immune complex deposition. Hum Pathol 2008;39:73-79.

22 Adeyi OA, Girnita AL, Howe J, et al. Serum analysis after transplant nephrectomy reveals restricted antibody specificity patterns against structurally defined HLA class I mismatches. Transpl Immunol 2005;14:53-62.

23 StataSoft I. STATISTICA for Windows [Computer Program Manual]. StatSoft Inc: Tulsa, OK, 1995.

24 Sun Q, Liu Z, Yin G, et al. Detectable circulating antiendothelial cell antibodies in renal allograft recipients with C4d-positive acute rejection: a report of three cases. Transplantation 2005;79:1759-1762.

25 Regele H, Böhmig GA, Habicht A, et al. Capillary deposition of complement split product $\mathrm{C} 4 \mathrm{~d}$ in renal allografts is associated with basement membrane injury in peritubular and glomerular capillaries: a contribution of humoral immunity to chronic allograft rejection. J Am Soc Nephrol 2002;13:2371-2380.

26 Celik B, Randhawa PS. Glomerular changes in BK virus nephropathy. Hum Pathol 2004;35:367-370. 